\title{
modalidades de interacción y valores sociales emergentes en niños*
}

\section{Interaction Modalities and the Emergence of Social Ualues in Children modalidades de interação e valores sociais emergentes em crianças}

\author{
Mónica Roncancio Moreno** \\ Universidad Pontificia Bolivariana \\ Danna Aristizábal Oviedo \\ Universidad Cooperativa de Colombia
}

Doi: http://dx.doi.org/10.12804/revistas.urosario.edu.co/apl/a.7965

\section{Resumen}

Los valores sociales han sido estudiados desde diferentes perspectivas. En la mayoría de investigaciones se intenta determinar que tan cooperativa o individualista es una sociedad. La presente investigación tiene como propósito identificar la emergencia de valores sociales en niños, durante un proceso de interacción entre pares, desde una perspectiva de la psicología sociocultural. Con el uso de una metodología cualitativa, se trabajó con 66 niños y niñas de tres ciudades colombianas que se enfrentaron a una situación semiestructurada que implicaba la construcción de un objeto (en solitario o conjuntamente). Todas las parejas fueron grabadas en video. Después del análisis microgenético (categorías emergentes), se identificaron cuatro modalidades de realización de la tarea, preinteraccional, interaccional-individual (convergente), interaccional-individual (ambivalente) e interaccional-cooperativa (convergente). Los resultados indican que la mayoría de las modalidades se centraron en el trabajo individual, privilegiando las metas personales de los sujetos con algunos momentos de cooperación. Se sugiere explorar a profundidad la dinámica de las relaciones entre pares en los contextos escolares.

Palabras clave: cooperación, individualismo, solidaridad, competición, perspectiva sociocultural, valores sociales.

\section{Abstract}

The social values have been studied from different perspectives. Most of researches, are conducted to determine how cooperative or individualistic a society is. The purpose of this research is to identify the emergence of social values in children during a process of peer interaction,

* Este artículo es producto de la investigación "Caracterización del sistema de valores en niños de 10 años a través de narrativas escritas”, financiada por la Universidad Cooperativa de Colombia (Radicado inv - 2382-I) y por la Universidad Pontificia Bolivariana-Seccional Palmira (Radicado 023A-07/18-02P).

** Dirigir correspondencia a Mónica Roncancio Moreno, Universidad Pontificia Bolivariana, Seccional Palmira. Correo electrónico: monica.roncancio@upb.edu.co

Cómo citar este artículo: Roncancio-Moreno, M., \& Aristizábal, O. D. (2019). Modalidades de interacción y valores sociales emergentes en niños. Avances en Psicología Latinoamericana, 37(3), 455-470. Doi: http://dx.doi.org/10.12804/revistas.urosario.edu. co/apl/a.7965 
from a sociocultural psychology perspective. We studied 66 children from three Colombian cities who faced a semi-structured situation that involved the construction of an object (alone or together). All couples were recorded on video. After the micro-genetic analysis (emergent categories), four modalities of accomplishment of the task were identified: Pre-interactional, Interactional-Individual (Convergent), Interactional-Individual (Ambivalent), and Interactional-Cooperative (Convergent). The results indicate that most of the modalities focused on individual work, privileging personal goals with some markers of cooperation. Researchers suggest exploring in depth the dynamics of peer relations in the school contexts.

Keywords: Cooperation, individualism, solidarity, sociocultural perspective, competition, social values.

\section{Resumo}

Os valores sociais tem sido estudados desde diferentes perspectivas. A maioria das pesquisas, tem a pretensão de determinar quanto uma sociedade é cooperativa ou individualista. A presente pesquisa tem como propósito identificar a emergência de valores sociais em crianças durante um processo de interação entre pares, desde uma perspectiva da psicologia sociocultural. Com o uso de uma metodologia qualitativa, se trabalhou com 66 crianças de três cidades colombianas que se enfrentaram a uma situação semiestruturada que implicava a construção de um objeto (em solitário ou conjuntamente). Todos os casais foram filmados em vídeo. Depois da análise microgenética (categorias emergentes) se identificaram quatro modalidades de realização da tarefa, pré-interacional, interacional -individual (convergente), interacional-individual (ambivalente) e interacional-cooperativa (convergente). Os resultados indicam que a maioria das modalidades se centraram no trabalho individual, privilegiando as metas pessoais dos sujeitos com alguns momentos de cooperação. Sugere-se explorar em profundidade a dinâmica das relações entre pares nos contextos escolares.

Palavras-chave: cooperação, individualismo, solidariedade, competição, perspectiva sociocultural, valores sociais.
Las investigaciones centradas en el estudio de valores prosociales, desde diferentes perspectivas, han sido un tema de gran interés desde la década del setenta (Brownell \& Carriger, 1990; Correa-Chávez, 2016; Kagan \& Madsen, 1971; Matusov, Bell \& Rogoff, 2002). Numerosas investigaciones se han centrado en la orientación del valor social (Social Value Orientation - Svo), la cual intenta establecer las diferencias individuales entre sujetos cooperativos e individualistas con el uso de diferentes medidas asociadas (Pletzer, Balliet, Joireman, Kuhlman, Voelpel \& Van Lange, 2018). La mayoría de estudios reportados intentan describir qué tanto un individuo o sociedad es individualista, cooperativa o competitiva (Pletzer et al. 2018). Este tipo de perspectivas, de corte positivista, intentan encontrar correlaciones entre comportamientos prosociales y problemas, así como también hacen uso de diversos dilemas sociales como herramienta metodológica (Hay \& Pawlby, 2003).

Por otro lado, existen perspectivas que profundizan en los significados involucrados en la emergencia de los valores sociales, como la perspectiva sociocultural (Valsiner \& Rosa, 2007; Branco \& Valsiner, 2012). El presente artículo se ubica en dicha perspectiva y se deriva de un macroproyecto de investigación $;^{1}$ su objetivo es identificar los marcos de comunicación durante una situación semiestrucurada en la cual emergen valores sociales.

\section{La perspectiva sociocultural y el estudio de los valores sociales}

Desde finales de la década del ochenta, se ha gestado un nuevo campo teórico denominado psicología sociocultural, el cual surge de la síntesis de

\footnotetext{
El macroproyecto "Caracterización del sistema de valores en niños de 10 años a través de narrativas escritas" profundizó en la co-construcción de valores como la cooperación, la solidaridad, el individualismo y la competencia, en niños de diferentes instituciones educativas públicas de dos regiones de Colombia.
} 
varias disciplinas como la psicología, antropología, sociología, historia y ciencias médicas (Branco \& Lopes de Oliveira, 2012; Valsiner \& Rosa, 2007; Valsiner, 2014). Desde este abordaje, se asume el desarrollo humano como un fenómeno sistémico, abierto y dinámico, que se transforma de manera constante. Se hace énfasis en el cambio en la trayectoria de vida de los sujetos y en la creación y emergencia de significados y valores sociales. La perspectiva sociocultural propone el diálogo y mutua constitución entre sujeto y cultura, reconoce la singularidad de los individuos y su carácter histórico-cultural (Valsiner, 2014). En este abordaje, se destaca la afectividad como principal elemento en el estudio del dominio psicológico en tópicos como creencias y valores sociales (Branco \& Valsiner, 2012). El interés de la presente investigación se centra en el estudio de los valores sociales.

Los valores sociales, desde la perspectiva sociocultural, son considerados como parte de los significados o sistemas semióticos que construyen los individuos y como un tipo de proceso psicológico diferenciado de las creencias, por su base fundamentalmente afectiva y su mayor resistencia a los cambios durante el desarrollo (Valsiner, Branco \& Dantas, 1997). Son un conjunto de afectos, cogniciones y motivaciones vinculados a las prácticas y experiencias que emergen durante el desarro1lo, que actúan como mediadores semióticos que alimentan las diferentes posiciones personales asumidas por un individuo (Hermans, 2001) en los diferentes contextos en los que se desenvuelve. En estos contextos, el individuo sitúa sus relaciones, le otorga un propósito a su vida y participa en la construcción de valores mediante la interacción con los otros (Branco \& Valsiner, 2012).

En específico, se afirma que el conocimiento relacionado con la construcción de sistema de valores es fundamental para comprender las motivaciones y acciones de los individuos. Por tanto, desde la perspectiva sociocultural, la psicología debe ocuparse de la identificación y comprensión de las dinámicas personales asociadas con la construcción conjunta de las prácticas socioculturales y los significados cargados de afecto que las personas elaboran sobre su identidad y su vida (Branco \& Valsiner, 2012; Marsico \& Varzi, 2015; Tateo, 2018).

El estudio de los valores como proceso psicológico es importante en tanto estos promueven acciones que contribuyen al desarrollo positivo de los individuos y los grupos, pero también pueden fomentar la aparición de niveles de destrucción y violencia (Rengifo-Herrera \& Branco, 2014). En este sentido, los valores pueden ser de tipo constructivo o no constructivo. Los primeros están relacionados con la promoción de acciones cooperativas, solidaridad y beneficio común; altruismo, protección y cuidado, por ejemplo. Los segundos incluyen acciones vinculadas con la exclusión, ataque, daño y falta de respeto hacia los intereses de los demás (Rengifo-Herrera \& Branco, 2014). Así, identificar el tipo de valores que los niños construyen, y que circulan en sus contextos más próximos, podrá permitir la elaboración de programas de prevención sobre violencia y bullying en la escuela, entre otros.

\section{La cooperación, la competición, el individualismo y la solidaridad}

Estudios previos (Branco, 1998; Maciel, Branco \& Valsiner, 2004) se han centrado en la exploración de los valores, por medio de la caracterización de su marco intersubjetivo, que puede ser de convergencia y divergencia. En el primero, se incluyen los valores de solidaridad y cooperación y en el segundo, los de individualismo y competición.

Desde la psicología sociocultural, la cooperación se define como la coordinación de metas entre individuos en situaciones específicas, en un marco intersubjetivo en el que se configura un contexto relacional que los individuos en interacción construyen y sirve como base para la interpretación de significados (Palmieri \& Branco, 2014). Los sujetos involucrados en la situación trabajan por 
una meta y las estrategias son acordadas y compartidas porque son útiles a los fines de todos. Por ejemplo, el niño o niña no necesariamente piensa en sus propias necesidades o en las necesidades del otro sino también en la meta común y en concertar acciones para alcanzarla.

Por su parte, la solidaridad es definida como el resultado del reconocimiento, respeto y consideración ante otros que posibilita que la persona se imagine a sí misma en una situación similar a la de otras personas (Branco, 2016). Cuando emerge la solidaridad, hay un reconocimiento de un objetivo común y el compromiso activo para su resolución. Se reconoce la posición del otro en términos de emociones y creencias, por lo que se acepta una estrategia de resolución que le sea útil al otro, y no necesariamente a uno mismo. En este caso, el niño o niña reconocen el problema que no necesariamente los involucra de forma directa, pero con el cual se sienten vinculados en alguna instancia. La estrategia de resolución, a pesar de no ser compartida, contribuye a solucionar el problema.

La competición se define como la orientación de las acciones hacia un objetivo que se considera de exclusivo cumplimiento, motivado por el deseo de mostrar desempeños superiores a los de los otros. En este caso, las personas trabajan por un objetivo exclusivo, sin considerar la posibilidad de una meta conjunta. Implica celebrar el propio éxito ante el fracaso de los demás y pueden verse implicados comportamientos hostiles. En este caso, los niños y las niñas se centran en que deben cumplir el objetivo por sí solos, con la intención de ser mejores que los otros.

El individualismo se define como las acciones del sujeto orientadas por la intención de cumplir una meta por sí solo, sin considerar el posible aporte de otros al proceso. Las personas trabajan por un objetivo individual, no se considera una meta conjunta pero tampoco implica que se presenten comportamientos hostiles. Los niños y las niñas se centran en un objetivo que no se considera compartido y prefieren resolver la situación por sí mismos.

\section{Los procesos de interacción y la emergencia de marcos comunicativos}

En los diferentes escenarios socioculturales, en los cuales los individuos interactúan, se crean marcos de comunicación y metacomunicación en los que emergen significados y valores sociales (Branco, Pessina, Flores \& Salomão, 2004; Glaveanu, Branco \& Neves-Pereira, 2016; Tacca, 2000). El proceso de interacción se genera en el marco de los procesos comunicativos y metacomunicativos que a su vez hacen parten de la dinámica imbricada de internalización y externalización. La comunicación, desde esta perspectiva, se define como el proceso de co-construcción de significados dinámico, que involucra el intercambio de una serie de signos y que se expresa mediante diferentes canales comunicativos (Branco et al. 2004). Por su parte, la metacomunicación se considera como una dimensión fundamental de la comunicación, se refiere a la comunicación sobre la comunicación y principalmente tiene que ver con la dimensión relacional de la comunicación, es decir, con las interacciones (Branco et al. 2004).

Por otro lado, en el contexto de las interacciones escolares, específicamente, el comportamiento observable cumple un papel central como base para inferir orientaciones de los sujetos acerca de sus objetivos. De igual forma, esas orientaciones pueden cambiar a lo largo del proceso de interacción, pues en general este no implica propósitos bien definidos. Para realizar esa descripción del comportamiento observable es necesario un análisis que vaya más allá de la identificación de las acciones y que se centre en las múltiples transformaciones ocurridas en el proceso de interacción, principalmente en la emergencia de significados (Branco, 2018).

En el marco de un proceso de interacción se generan niveles de organización de las acciones y la coordinación de metas de los individuos, en relación con la tarea o con la situación, que dan lugar a la emergencia de valores de cooperación, 
solidaridad, competición e individualismo. Se puede inferir el tipo de valor que emerge a partir de la compatibilidad de las orientaciones a objetivos sobre la base de las acciones de los sujetos en interacción. Asimismo, es posible identificar la calidad de los marcos de comunicación durante ese proceso: convergentes, divergentes y ambivalentes (Maciel et al. 1998).

\section{Metodología}

La presente investigación se inscribe en una perspectiva idiográfica (Salvatore \& Valsiner, 2010) y microgenética (Barrios, Barbato \& Branco, 2012). Desde la perspectiva idiográfica, se reconoce el carácter único de las experiencias de los sujetos y el principal abordaje es el estudio de caso. Desde lo microgenético, se pretende recuperar la emergencia de los significados en el marco de los procesos de interacción.

\section{Participantes}

Tomaron parte 66 participantes entre los $10 \mathrm{y}$ los 11 años (49 niñas y 17 niños) de tres ciudades diferentes de los departamentos de Valle del Cauca y Risaralda, Colombia. En la ciudad de Cali participaron 12 parejas, en la ciudad de Palmira, 11 y, finalmente, en La Virginia, 10. Los participantes pertenecían a colegios públicos de cada una de las ciudades y cursaban quinto grado de básica primaria. La selección de las parejas se realizó de manera aleatoria. Pertenecían al mismo salón y habían compartido como compañeros de curso por lo menos ocho meses. Se realizaron parejas tanto de niñas como de niños y mixtas, pero predominaron las de niñas, debido a la gran cantidad de estudiantes de sexo femenino en los grupos.

\section{Procedimiento}

Después del contacto con las instituciones educativas, tanto los padres como los niños y niñas firmaron consentimientos informados. Todos los nombres de los niños y las niñas fueron modificados para mantener la confidencialidad.

\section{Situación semiestructurada}

Se diseñó una situación semiestructurada a resolver entre dos participantes. La situación tenía como objetivo elaborar un juguete o algún objeto en parejas de niños y niñas. El propósito era que, en el camino para la resolución de este objetivo, los niños y las niñas interactuaran y crearan marcos de comunicación, de convergencia o de divergencia, en los cuales emergerían los valores sociales.

Se dispuso un kit compuesto por minibloques de madera, felpillas para manualidades, palillos, una hoja, un lápiz y pegante. Se le presentaba a la pareja los materiales y se le daba la siguiente consigna: "ustedes dos tendrán estos materiales para construir lo que quieran. Deben indicar cuando hayan acabado". El objetivo era dejar la consigna lo suficientemente abierta para que los participantes decidieran espontáneamente trabajar juntos o de manera individual. En caso de que preguntasen qué tipo de objeto se debía realizar, las investigadoras sugerían un juguete, un muñeco, etc. Se les permitía usar todo el tiempo necesario para construir su objeto. Una vez indicaban que habían terminado, se les decía: "ahora, deben decidir quién se quedará con lo que han construido". Tomaban la decisión de forma individual, sin la intervención de las investigadoras. Esta última parte de la actividad era fundamental para conocer si los participantes realizaban una negociación por sí solos o si requerían la intervención del adulto para decidir. Toda la situación fue grabada en video, la cámara enfocaba el plano en el cual se ubicaron y registraba lo que los niños decían y hacían, mientras que un miembro del equipo de la investigación respondía a dudas o inquietudes de los niños y niñas, el otro grababa. 


\section{Análisis de la información}

Una vez recolectados los videos, se realizó el análisis de las situaciones por medio del método microgenético, desde una perspectiva sociocultural (Barrios et al. 2012). Por acuerdo entre investigadores, se segmentaron los videos en episodios y se clasificaron en relación con el tipo de marco de comunicación, interaccional o preinteraccional (Lordelo \& Carvalho, 2006; Pedrosa \& Carvalho, 2006). Los marcos interaccionales se clasificaron como cooperativos o individualistas. Finalmente, se clasificó la calidad del marco comunicativo como convergente, divergente o ambivalente.

Se pretendía analizar la dinámica de las interacciones sociales entre los sujetos participantes de la situación semiestructurada. Para el análisis, se definieron tres características: 1) el nivel de los aspectos estructurales de las interacciones (interacción o preinteracción); 2) el nivel de la coordinación de las metas (individual, competitiva, cooperativa) y 3) la calidad de los marcos comunicativos (cooperativa, competitiva o individual).

En los aspectos estructurales de las interacciones, se definieron dos niveles de organización de las acciones: preinteraccional e interaccional. Existe preinteracción cuando un sujeto invita al otro a la interacción verbalmente o físicamente, pero el otro no responde ni se involucra en un intercambio comunicativo. En estos casos, un individuo despliega una acción hacia el otro, lo mira o lo invita jugar, pero el otro no responde a la acción del compañero. También se clasifican observaciones e imitaciones en las cuales la participación del otro no se detecta o cuando ninguno de los participantes despliega acciones que muestren intención de interacción. De igual forma, puede que haya o no una intención de interacción que al final no se concrete. Por ejemplo, una de las niñas le habla a la otra o la invita a construir el objeto de manera conjunta, pero el otro niño o niña lo ignora.

Por otro lado, se define el nivel interaccional en el cual existe intersubjetividad y los participantes despliegan movimientos sociales de los unos con los otros. En este tipo de marcos se toma en cuenta el contenido de los significados en la dimensión de los encuentros sociales entre individuos y es posible diferenciar el flujo interactivo en patrones específicos. En este nivel se coordinan las acciones hacia la meta, ya sean estas cooperativas, competitivas, individuales o solidarias. A partir de la coordinación de las metas se puede estudiar la calidad del marco comunicativo de los sujetos, sea esta convergente, divergente o ambivalente.

Un marco de convergencia se crea cuando existe compatibilidad de las orientaciones hacia los objetivos, lo que significa que no es esencial que las personas busquen juntos el logro de la meta. Por ejemplo, dos sujetos pueden estar realizando una tarea de manera individual, pero, al mismo tiempo, pueden estar interactuando de manera recíproca, compartiendo significados y facilitándose entre ellos el alcanzar la meta. La convergencia crea una base relativamente estable para los esfuerzos de comunicación y conduce a una cierta consistencia en la interacción que permite la construcción activa de la intersubjetividad entre los participantes, es decir, las acciones de los niños se organizan alrededor de un objetivo compatible para todos (Branco, 1998).

Por otro lado, existe divergencia cuando los objetivos que pertenecen a cada individuo son incompatibles, es decir, no pueden lograrse al mismo tiempo. Por ejemplo, dos niños están trabajando juntos en la construcción de un objeto, pero compiten por terminarlo o por quién lo hace mejor. La divergencia puede crear las condiciones para el surgimiento de la novedad y, en consecuencia, puede dar lugar a una negociación, lo que facilita la emergencia de nuevos patrones de interacción (Branco, 1998). Existe ambivalencia cuando aparece involucramiento positivo en la actividad con situaciones de hostilidad, pero se mantiene una dinámica de reciprocidad.

De acuerdo con los niveles de organización estructural preinteraccional e interaccional, la 
coordinación de las metas y la calidad de los marcos comunicativos, se identificaron cuatro modalidades de realización de la tarea:

\section{Preinteraccional}

2. Interaccional-Individual (Convergente)

3. Interaccional-Individual (Ambivalente)

4. Interaccional-Cooperativa (Convergente)

\section{Resultados}

Los resultados de esta investigación se presentarán en dos secciones. Inicialmente, se carac- terizarán los casos de cada una de las ciudades que participaron en el estudio (tabla 1 y figura 1). Posteriormente, se describirán las características de las cuatro modalidades de realización de la tarea.

Como se puede observar en la tabla 1 y en la figura 1 , la modalidad de realización preinteraccional fue la que más predominó en todas las regiones con un $67 \%$, seguida de las modalidades Interaccional-Individual (Convergente) e Interaccional-Cooperativa (Convergente) con un $12 \% \mathrm{y}$, finalmente, la Interaccional-Individual (Ambivalente) con un 9\%.

Tabla 1.

Modalidades de realización por las tres ciudades

\begin{tabular}{cl}
\hline Ciudad & \multicolumn{1}{c}{ Resultados } \\
\hline \multirow{2}{*}{ Cali } & $\begin{array}{l}\text { En total, se realizaron } 12 \text { situaciones semiestructuradas en Cali y un total de tres horas y 53 minutos de } \\
\text { grabación de video. Se identificaron las cuatro modalidades de realización, distribuidas de la siguiente } \\
\text { forma: ocho modalidades preinteraccionales, dos interaccionales-individuales (Convergente), una } \\
\text { interaccional-individual (Ambivalente) y una interaccional-cooperativa (Convergente). }\end{array}$ \\
\hline \multirow{2}{*}{ Palmira $\quad \begin{array}{l}\text { En total, se realizaron 11 situaciones semiestructuradas y se registraron tres horas y dieciséis minutos de } \\
\text { grabación de video. Se identificaron ocho modalidades preinteraccionales, dos interaccionales-individuales } \\
\text { (Ambivalente) y una interaccional-cooperativa (Convergente). }\end{array}$} \\
\hline \multirow{2}{*}{ La Virginia } & $\begin{array}{l}\text { En La Virginia se realizaron 10 situaciones semiestructuradas para un total de tres horas y 59 minutos } \\
\text { de grabación de video. Se identificaron seis modalidades de realización preinteraccionales, dos } \\
\text { interaccionales-individuales (Convergentes) y dos interaccionales-cooperativas (Convergentes). }\end{array}$ \\
\hline
\end{tabular}

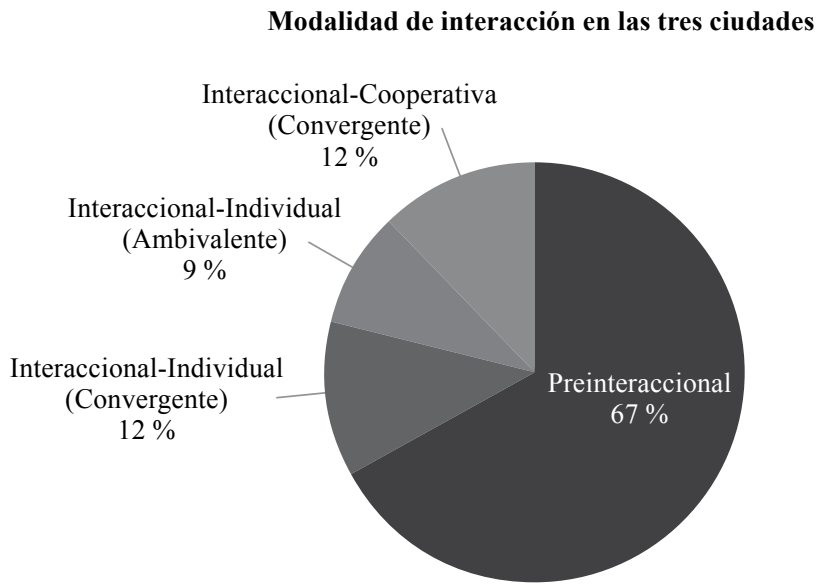

Figura 1. Distribución de las modalidades por ciudad 


\section{Análisis de las modalidades de realización de la tarea}

Las modalidades reportadas indican que, a pesar de que predominan ciertos procesos de interacción o de preinteracción, existe una dinámica que puede derivar en momentos de negociación o de emergencia de otros valores sociales. Para la presentación de los casos, se tomará un caso típico que ilustrará la modalidad de realización de la tarea. Se definieron diferentes episodios a partir del recorte de los momentos de intercambio entre los sujetos.

\section{Modalidad de realización preinteraccional}

Esta modalidad fue la más común en todas las regiones, con un total de 22 casos. Se caracteriza por privilegiar el trabajo individual, sin tener en consideración al compañero de actividad. La mayoría de los niños realizaban la construcción del objeto en solitario e, incluso, en algunos momentos reñían o no lograban ponerse de acuerdo en la distribución de los materiales para la realización de la actividad.

Sin embargo, a pesar de que estas modalidades son principalmente preinteraccionales, existen algunos momentos en los cuales se presentan marcos comunicativos con convergencias o divergencias. Las preinteracciones tuvieron dos características, primero, una de las participantes intentaba iniciar la interacción con miradas, sonrisas e incluso verbalizaciones, pero no encontraba retorno en su compañera de actividad. En algunos momentos, una de las participantes pedía ayuda, pero la otra parecía no escucharla. Segundo, los participantes no establecieron contacto o intento de comunicación entre ellos.

En este tipo de modalidad, los niños no se orientaron a la meta de manera conjunta ni negociaron los objetivos de la tarea, pero sí se esforzaron por cumplir la meta de la actividad de manera individual. De igual forma, en este tipo de modalidad, emergieron valores de competición.
Por ejemplo, Juana y María ${ }^{2}$ desarrollaron la actividad de manera individual, si bien en algunos momentos emergieron interacciones caracterizadas por miradas y sonrisas, no apareció conversación. Hacia el final de la actividad, cuando se les pidió a las niñas realizar negociaciones sobre quién se quedaría con los objetos, se encontró que ambas niñas querían quedarse con ellos (con todo) y una de ellas buscó conservarlos de manera insistente, como se evidencia en el siguiente fragmento de la situación.

\section{Episodio 20}

Investigadora: ¿Quién se va a llevar los objetos? Juana: yo me lo quiero llevar.

María: yo me lo quiero llevar (énfasis).

Investigadora: ¿tú te lo quieres llevar y tú te lo quieres llevar?

Ambas asienten

Investigadora: bueno, van a tomar una decisión María: ¿cómo así?

Investigadora: de quien se lleva lo realizado.

María voltea a mirar a Juana y le dice algo en un tono muy bajo (inaudible) y añade: ¿sí? (Juana tiene su mirada en la mesa y no contesta).

María le dice a Juana ¿me lo llevo? Juana la mira, María le dice de nuevo: “¿me lo llevo?” Juana desvía la mirada de María y vuelve a mirar la mesa sin contestar.

María mira la mesa y vuelve la mirada a Juana y le dice ¿me lo llevo? Juana le contesta: sí

María: ¿sí?

Juana asiente mientras la mira y ambas voltean al frente a mirar a la investigadora y María le dice sonriente a la investigadora: ¡sí me lo voy a llevar! (contenta)

Investigadora: ¿cómo?

María sonríe y dice: ¡me lo voy a llevar!

Investigadora: ¿tú te lo vas a llevar?

María asiente con la cabeza.

Investigadora: ¿y te lo vas a llevar todo?

Se cambiaron los nombres de los participantes por razones éticas. 
María asiente nuevamente.

Investigadora: listo (le acerca los objetos a María).

María sonríe mientras coge los objetos construidos y Juana, con rostro inexpresivo, intercala la mirada entre lo que hace María, la investigadora y la cámara. María mira por un momento a Juana, pero vuelve su vista los objetos y después a la investigadora.

En esta pareja de participantes se observó que la preinteracción permanece prácticamente hasta el final. Hacia el final, cuando la investigadora solicita la definición de quién se quedará con el juguete — parte de la actividad que fue diseñada esencialmente para que los niños negocien — ambas niñas manifiestan su interés en tenerlo y se desencadena un episodio en el cual una de las niñas insiste en quedarse con él ante la indiferencia de la otra.

Este episodio muestra cómo Juana, quien también declara que quiere el objeto, deja que María se lo lleve sin hacer ningún intento para quedarse con él. Esta última parte de la situación está diseñada para que emerjan negociaciones entre los niños, pero en esta pareja se observa que se mantiene el marco preinteraccional.

\section{Modalidad interaccional-individual (convergente)}

En esta modalidad se registró un total de cuatro casos en las tres ciudades. Esta modalidad se caracteriza por la elaboración del objeto de manera individual (coordinación de la meta), pero con interacciones constantes entre las participantes en un marco comunicativo convergente. Las participantes se involucraron en la actividad y cooperaron tanto en el diálogo como en la acción. Se encontró que una de las participantes observaba lo que la otra compañera estaba creando.

En el caso de Camila y Angely, se observó desde el inicio de la actividad que las niñas acuerdan qué van a construir, aunque en el transcurso del ejercicio y del intercambio transforman lo que inicialmente habían propuesto. En este proceso aparecen momentos en los que las participantes se dan instrucciones entre ellas, por ejemplo, "[...] y haces la forma de una hojita con verde", "puedes ponerle una naricita", "está bien yo hago un conejito" o en los que se piden ayuda y aparece colaboración entre ellas ("¿cómo hago para hacer...?"), encontrando siempre reciprocidad. De igual forma, durante la actividad, las participantes aplauden ante el logro de la otra compañera (tras haber realizado parte del objeto) y comparten información sobre su cotidianidad.

A pesar de que esta modalidad se clasifica como individual, en términos de coordinación de las metas, se puede afirmar que ambas participantes se articularon mutuamente para cooperar y alcanzar un objetivo común. Este marco comunicativo se caracteriza por la convergencia, se observa confianza y expresiones de afecto entre ellas. En el episodio 35 se observa la última parte de la actividad, el momento en el cual las participantes deben decidir quién se quedará con el objeto.

Episodio 35

Entonces deben escoger ¿quién se va a quedar con los objetos que hicieron?

Camila: [dirigiéndose a Angely] ¡yo! (énfasis)

Inv: ¿con todo?

Camila: ¡con todo! (énfasis, seguridad)

Angely sonríe.

Camila le dice a Angely algo en el oído.

Angely dice: sí, que se lo quede ella.

Cuando la investigadora les pregunta quién se quedará con el objeto, no se observa negociación, por el contrario, es Camila quien enfáticamente decide que será ella y, durante el proceso, se dirige a Angely en "secreto", ante lo cual Angely cede y dice "sí, que se lo quede ella". Esta última parte de la actividad da cuenta de la característica convergente del marco comunicativo de las participantes. 


\section{Modalidad de realización interaccional individual (ambivalente)}

Esta modalidad se caracteriza por un nivel de organización interaccional con una coordinación de acciones individual, pues ambos participantes favorecen la realización del objeto en solitario. La calidad del marco comunicativo es ambivalente, pues presenta tanto momentos de cooperación como de hostilidad.

En esta modalidad se observan diferentes momentos de convergencia y negociaciones, a partir del diálogo, y algunos momentos de hostilidad. Desde el inicio, las participantes se involucraron en la actividad, mostrando disposición e interés en el proceso de construcción del objeto. Los momentos de convergencia y cooperación emergieron en casi todo el proceso de elaboración de los objetos. En la conversación, las niñas cooperaron en el diálogo e intercambiaron experiencias sobre el objeto que cada una de ellas estaba construyendo por separado.

En el episodio 1, se observa cómo ambas niñas se involucraron en la actividad y cómo el intercambio comunicativo es afectivo. El episodio 1 es un ejemplo de los múltiples momentos en los cuales se presentó cooperación por medio del diálogo de las dos participantes.

\section{Episodio 1}

Daniela dirige su mirada hacia Luisa, esta última no la mira, pero se ríen al mismo tiempo, Daniela se voltea y mira la investigadora riéndose con ella, Luisa se ríe fuerte, mientras que Daniela mete su mano a la bolsa y dice en voz alta: "un carro grande". Luisa empieza a reírse con mucha más intensidad y Daniela se ríe también, mira a la investigadora y dice: "Luisa me hace dar risa" y vuelve su mirada hacia su construcción. En medio de risas, Luisa mira la construcción de su compañera y le dice: "no pude, Daniela". Daniela acomoda su construcción, mira la investigadora y sonriendo exclama: “¿eso va pa' un lado o pa' otro", Luisa le muestra su construcción a Daniela mientras se ríe, Daniela se ríe con ella y le pregunta: ¿Uy, eso qué es? Ambas niñas se ríen al mismo tiempo y en un tono alto, Daniela dice: "una trenza" y mira a su compañera, la cual, en medio de risas, le contesta: "nooo, es que no lo puedo hacer bien". Ambas niñas se ríen.

[...] Luisa le muestra a Daniela su construcción y le dice: "Ay, sí la pude hacer, ve" [emoción], a lo que Daniela le contesta: "ay, sí" [emoción].

[...] Luisa dirige su mirada hacia Daniela y con la construcción que hizo toca la cara de su compañera con el fin de hacerle cosquillas. Luisa reacciona y se corre hacia un lado, luego de esto se ríe.

[...] Daniela le dice a Luisa: "tan estúpida" y de inmediato dirige su mirada hacia la investigadora. Luisa sigue concentrada en su construcción.

Episodio 8

Luisa le muestra a su compañera la construcción que ha realizado y le dice: "este es mi gusano", Daniela lo mira, se ríe y contesta: "pues el gusano no se hace así", coge uno de los escobillones y lo empieza a doblar. Luisa exclama: "no, pero el mío va a ser..."

En el episodio 1 emerge un momento de hostilidad cuando Daniela le dice a Luisa "tan estúpida", ante las cosquillas que le hace la compañera. En la interacción entre estas dos participantes se evidenció una riqueza en el diálogo y en la afectividad que se crea en el marco comunicativo y en ese intercambio - que la mayoría de las veces está caracterizado por la reciprocidad - en el que también emergen momentos de hostilidad. Los momentos de hostilidad no modifican la relación que se puede inferir entre las dos participantes, pues estos van seguidos de interacciones convergentes y de negociaciones, lo que indica que el marco de comunicación es "ambivalente". Uno de los momentos de hostilidad fue caracterizado por la comparación entre los dos objetos. Por otro lado, a pesar de que las participantes desarrollan la 
actividad de manera individual, cooperan permanentemente y negocian la orientación a objetivos de la tarea.

Otro ejemplo de la modalidad individual convergente es el caso de Juliana y Valeria, caracterizado por una gran riqueza en los procesos de interacción de ambas participantes. En esta pareja se observa la emergencia de dos momentos de cooperación, al inicio de la actividad cuando cada una decide qué van a diseñar en común acuerdo con la otra. Posteriormente, se identifican varios momentos en los cuales las niñas corresponden en miradas y sonrisas aprobando la actividad diseñada por cada una de ellas.

En el intercambio entre estas dos participantes es posible ver variabilidad en los diferentes momentos de interacción y el tránsito entre interacciones convergentes y divergentes (episodios 5 y 8), lo que da cuenta de un marco comunicativo ambivalente.

\section{Episodio 5}

Valeria le pregunta a Juliana "¿el pegamento pega bien?", Juliana le responde “jum, ay, yo no sé", al mismo tiempo Juliana mueve arriba y abajo su mano izquierda, seguidamente le dice a Valeria "¿y qué? es que me cogió el corazoncito y yo ahora ¿qué voy a pegar? tan atrevidita (en tono sarcástico y con molestia)".

\section{Episodio 8}

Juliana estira su mano y coge el pegamento que tiene Valeria sobre la mesa y le dice "pásame, no te la comas sola, no te la comas toda vos sola", Valeria no le responde nada ni la mira.

La dinámica de interacción de esta pareja resultó interesante en tanto da cuenta de momentos de hostilidad, seguidos por momentos de convergencia o incluso pedidos de ayuda de una de las participantes, que fueron ignorados por su compañera la mayor parte del tiempo. Se observó que una de las participantes, Juliana, era quien dominaba la actividad y precisamente quien tenía comportamientos de hostilidad con su compañera.

\section{Modalidad de realización interaccional- cooperativa (convergente)}

Esta modalidad se caracteriza por la coordinación de metas entre los participantes con una calidad de marco comunicativo convergentes. Ambos participantes cooperan para alcanzar la meta de la tarea. En total, se identificaron cuatro casos en las tres ciudades.

En esta pareja de niñas, Laura y Valentina, se observa que las participantes trabajan conjuntamente e interactúan convergentemente la mayor parte del tiempo, ya que las participantes tienen una interacción efectiva y colaboran mutuamente. Después de que la investigadora les diera la consigna a las participantes, Valentina pregunta si puede hacer "lo que quieran". Ante la respuesta afirmativa de la investigadora, la niña comienza a sacar los materiales y le da instrucciones a su compañera Laura sobre los elementos que requieren para realizar la actividad (ver episodio 1).

\section{Episodio 1}

Valentina saca un cubo, le pasa la bolsa a Laura y le dice "saca otro cuadro". Laura saca un cubo de la bolsa y se lo pasa a Valentina.

Valentina le dice a Laura “¿Cómo lo vamos a hacer?" Laura la mira y sonríe.

Valentina le dice a Laura: "saca los palitos". Laura saca los palitos de la bolsa y los pone al alcance de Valentina.

Valentina comienza a manipular los palitos y le muestra Laura cómo podrían acomodarse (sonríen).

Valentina le pregunta a la investigadora: “ ¿se pueden pegar los palitos con pegante?” La investigadora responde: "sí".

Laura saca el pegante de la bolsa y se lo entrega a Valentina.

Valentina comienza a pegar los palitos. Laura la observa. 
Valentina le dice a Laura: "ayúdame a sacar los ojos". Laura saca los ojos de la bolsa y otros materiales.

Con la ayuda de Valentina, Laura saca todos los materiales de la bolsa.

Valentina le pregunta a Laura "¿Qué hacemos?" Laura hace un gesto de no saber. Valentina le dice a Laura: “esto, ¿no?” (alejando los palitos del lugar donde estaban ubicados y haciendo una figura con ellos). Laura hace un gesto de no saber. Valentina entonces le dice: "como en todo el centro el muñeco" (manipulando algunos de los materiales).

Laura manipula algunos materiales y Valentina se interesa mirándolos.

Episodio 3

Laura le muestra Valentina un palito con un ojo y Valentina le dice: "sí" (pasa a realizar con un palito lo mismo que Laura). Durante el proceso, Laura cambia la propuesta poniendo una bolita en el palito además del ojo (se lo muestra a Valentina). Valentina dice: "lo voy a hacer así también".

Laura termina el muñeco y se lo muestra a Valentina. Valentina mira su propio muñeco y al no estar igual al de Laura se lo entrega. Laura entiende que debe completarlo para que quede igual que el de ella y lo hace.

Episodio 15

Laura le entrega a Valentina los palitos y Valentina intenta unirlos con los palitos con los ojos; Laura la ayuda y le da indicaciones para que puedan hacerlo. Laura toma uno de los palitos y le aplica pegamento, Valentina la observa y toma el otro palito para también hacer lo mismo.

Episodio 19

Valentina le dice a Laura "los cubitos" y ambas toman cubitos para anexarlos al juguete. Laura le propone a Valentina ponerle bolitas, Valentina la observa, le entiende la idea y le propone algo para que puedan hacerlo mejor, le muestra cómo hacerlo y la Laura lo hace como lo sugiere Valentina. Ambas se ayudan.

\section{Episodio 30}

Ambas niñas (sin comunicarse verbalmente) empiezan a buscar qué más ponerle a la pirámide, Laura toma una bolita rosada pequeña y la pone en la punta de la pirámide, Valentina la observa; Laura le muestra a Valentina una bola más grande de color morado (sonriendo), Valentina la mira (sonriendo) y la deja poner la bola grande en la punta de la pirámide. Laura ve que la bola es muy grande y la retira, mientas tanto Valentina trata de ubicar unas bolas más pequeñas que la propuesta por Laura a los lados de la pirámide.

\section{Episodio 45}

Investigadora: “¿quién se quedará con el objeto?

Ambas niñas se miran y sonríen; rápidamente Valentina dice "ella", ambas sonríen, Valentina dice "usted y señala a Laura", Laura dice "usted" (sonriendo), Valentina dice "no" y hace un gesto de negación, mira al suelo y sonríe. Valentina se ríe y mira a Laura y está también se ríe. Ambas se miran.

[...] Valentina se pone la mano en la boca y se queda en silencio mientras tanto Laura la mira y sonríe; Valentina le dice a Laura: “cójalo usted", Laura dice "usted" y Valentina dice "nooooo" y niega con la cabeza (ambas se ríen). Laura mira al suelo y dice "cójalo usted", Valentina niega con la cabeza, Laura se ríe.

Valentina le dice a la Laura "usted" y Laura sonríe. Laura mira a la investigadora encargada de la filmación y le dice "lo coge ella" mientras señala a Laura, la investigadora le pregunta ¿por qué? (Valentina se ríe y Laura la mira y se ríe también). La investigadora le dice ¿Por qué crees tú que tiene que ser ella?, Valentina dice "nooo, porque [...] (se ríe y se tapa la boca) $[. .$.$] porque a mí se me$ desbarata". La investigadora le pregunta a Laura ¿tú estás de acuerdo en quedarte con el juguete? (Laura mira a Valentina), Laura sonríe y dice "sí". 
Desde el inicio, las participantes se involucraron con el objetivo de la tarea. Valentina tomó la iniciativa al tener en cuenta a su compañera en dos momentos en los que dice: “¿Qué vamos a hacer? ¿Qué hacemos?”. Ambas deciden en el diálogo y en las acciones directamente que van a trabajar en conjunto y se coordinan para alcanzar la meta de la tarea. Los marcos comunicativos que emergen en la interacción son convergentes $\mathrm{y}$ es evidente que ambas niñas cooperan tanto en términos del diálogo como de las acciones orientadas al cumplimiento de la meta. Durante toda la actividad emergen momentos como los reportados en los episodios 15 y 19, en los cuales negocian la construcción del objeto y se evidencia reciprocidad entre las participantes. Las formas de involucrarse en la tarea variaron entre ellas. En algunos momentos, Laura asumía el liderazgo y en otros era Valentina quien lo hacía, sin dejar de cooperar en un ambiente de confianza. Se podría afirmar que la calidad de los marcos comunicativos fue positiva, marcada siempre por comportamientos afectuosos y de confianza entre las participantes, orientados al cumplimiento de la meta. En ninguna parte de la interacción, que duró más de 30 minutos, se presentaron momentos de hostilidad entre las participantes.

En el momento en el cual se les pide a las participantes decidir quién se quedará con el objeto (episodio 45), ambas niñas consideran que lo mejor es que la otra lo conserve. Esta situación es coherente con el resto de la actividad, en la cual emergió cooperación. En este último punto, Valentina y Laura demostraron interés la una en la otra, independientemente de sus propios deseos, lo que hizo posible la emergencia del valor de la solidaridad.

\section{Discusión}

En la presente investigación se exploró la emergencia de valores sociales, tales como cooperación, solidaridad, individualismo y competición, en parejas de niños y niñas durante un proceso de interacción al enfrentarse a una situación semiestructurada en la cual los participantes debían construir un objeto. Se parte del supuesto de que la emergencia de los valores sociales se puede dar en un proceso de interacción y que, además, se enmarcan en procesos de comunicación y metacomunicación caracterizados por una determinada calidad afectiva (Branco et al. 2004).

Se definieron tres criterios para la construcción de las modalidades de realización: nivel de organización estructural (preinteraccional e interaccional), la coordinación de las metas (si el objeto se realizaba individualmente o conjuntamente) y la calidad de los marcos comunicativos (convergente, divergente o ambivalente). La clasificación de las modalidades de realización permitió enmarcar las diferentes dinámicas microgenéticas de las interacciones sociales de los niños participantes, lo que es coherente con estudios desarrollados desde perspectivas socioculturales del desarrollo (Branco, 1998; Glaveanu et al. 2016; Maciel et al. 2004; Palmieri \& Branco, 2014).

La clasificación de las modalidades, además, permitió exponer la riqueza de los procesos de interacción en los cuales emergen los valores sociales. Al propiciar una situación en la cual los niños decidían sobre trabajar por una meta conjunta o no, se favorecía una dinámica en la cual surgieron valores de diversa naturaleza, por ejemplo, en la interacción de una misma pareja emergieron significados relacionados con la cooperación, pero también el individualismo. De igual forma, en una de las parejas, cuya dinámica se caracterizó por el individualismo, en la última parte de la actividad emergieron significaciones relacionadas con la solidaridad. Esto indica que no es posible clasificar a un individuo como cooperativo o individualista, sino que diversos factores - como por ejemplo la calidad de los marcos comunicativos - estarían involucrados en la emergencia de los valores sociales. Lo anterior difiere de los estudios en los cuales se realizan clasificaciones sobre si los sujetos están 
más orientados por las metas personales (individualismo) o por las metas compatibles con la colectividad (cooperación) (Deutsch, 2015; Molleman \& Gachter, 2018; Pletzer et al.2018). No obstante, se relaciona con propuestas clásicas sobre el estudio y caracterización de los significados que emergen en las interacciones (Rengifo-Herrera \& Branco, 2014; Lottman, 2005) y que se acercan al marco epistemológico y conceptual sobre el que se desarrolló este trabajo.

En cuanto al nivel de organización estructural, se observó que la mayoría de los niños se ubicaron en una modalidad preinteraccional, lo que pone en evidencia que más de la mitad de los participantes no se interesaron en el intercambio con su compañero. Los grupos de participantes por ciudad correspondían al mismo salón de clases, por lo tanto, se realizó una selección aleatoria de las parejas y se consideró que todos los niños del grupo habían pasado más de ocho meses del año escolar juntos y que probablemente se les facilitaría la interacción. Se podría inferir que el elevado número de parejas con este tipo de modalidad corresponde con los valores promovidos en la escuela, los cuales tienen una base individualista (Borges \& Branco, 2019). De acuerdo con Branco y Valsiner (2012), los valores son internalizados por los sujetos a partir de la cultura colectiva y por medio de las experiencias de vida. Los valores sociales, como signos, se pueden expresar en términos de cooperación, competición, individualismo, hostilidad, violencia, etc. (Branco \& Valsiner, 2012). $\mathrm{Y}$, fundamentalmente, en función de su contexto.

En cuanto a la coordinación de las metas, una vez más, la mayoría de los participantes se orientaron a alcanzar un objetivo individual, expresado en la realización del objeto por separado. Sin embargo, algunos participantes cooperaron por medio del diálogo e, incluso, se presentaron casos en los cuales un niño ayudaba a otro a terminar su objeto. Se crearon marcos comunicativos en los cuales emergieron expresiones de afecto entre los participantes y, a pesar de que, como es el caso de los marcos ambivalentes, se presentaron momentos de hostilidad, estos venían seguidos de situaciones de cooperación. Lo anterior indica que la participación en actividades con interacciones convergentes acaba llevando a relaciones afectivas constructivas y a la emergencia de valores prosociales, como la cooperación y la solidaridad. Por otro lado, a pesar de que un marco comunicativo sea divergente, es posible que genere actividades cooperativas, probablemente con una calidad afectiva inferior a la de los marcos convergentes.

Para finalizar, en relación con lo metodológico, el diseño de una situación en la cual los niños podían decidir las diferentes formas de resolver la consigna permitió mayores posibilidades de acción de los participantes y de emergencia de los significados que configuran los valores sociales, en contraposición a los estudios que usan instrumentos como dilemas sociales - metodología ampliamente extendida en este tipo de estudios (Dawes, 1980) — L La situación semiestructurada permitió el intercambio comunicacional, la reciprocidad entre los participantes y la emergencia de momentos de cooperación entre pares.

Sin embargo, en futuros estudios, centrados en la emergencia de valores sociales, se hace necesario incluir, además, la contextualización de las interacciones en el ámbito de las relaciones humanas. Esto implica tener un conocimiento amplio de los participantes y de las dinámicas de relación construidas con sus pares, en este caso, en el contexto escolar. Las interacciones se enmarcan en las relaciones más amplias que tienen una determinada calidad afectiva y una dinámica de transformación en el tiempo, por lo tanto, es necesario explorarlas en profundidad. Se sugiere, entonces, realizar caracterizaciones de los contextos escolares y realizar un seguimiento de las relaciones de los participantes con su grupo de pares. 


\section{Agradecimientos}

Las autoras del artículo quieren agradecer al equipo de asistentes de investigación del proyecto pertenecientes a semilleros de investigación de las dos universidades, Duvan Darío Urbina, Angie Patricia Campo, Daniela Gutiérrez, Eliana Posso, Estefany Trujillo, Karen Mañunga, Alba Lucía Salazar, Harold Castañeda, Ingrid Perdomo, María Alejandra Ospina, Laura Echeverry, Laura Potosí, Alied Montenegro y Diana Vásquez.

\section{Referencias}

Barrios, A., Barbato, S., \& Branco, A. (2012). El análisis microgenético para el estudio del desarrollo moral: consideraciones teóricas y metodológicas. Revista de Psicología, 30(2), 249-279.

Borges, T., \& Branco, A. (2019). The production of school violence from a cultural psychology perspective. Estudios de psicología, 40(1), 107-133.

Branco, A. (1998). Cooperation, competition, and related issues: A co-constructive approach. In M. C. Lyra \& J. Valsiner (Eds.) Child development within culturally structured environments. Vol. 4. Construction of psychological processes in interpersonal communication (pp. 181-205). Norwood, N.J.: Ablex Publishing Corporation. Branco, A. (2016). Values and their ways of guiding the Psyche. In G. Marsico, J. Valsiner, N. Chaudhary, T. Sato, \& V. Dazzani (coords.), Psychology as the science of human being: The Yokohama manifesto (pp. 225-244). Switzerland: Springer.

Branco, A. (2018). Values, education and human development: The major role of social interactions' quality within classroom cultural contexts. In: A. U. Branco \& M. C. Lopes de Oliveira (Orgs.), Alterity, values, and socialization: human development within educational contexts (pp. 31-50). Cham: Springer.
Branco, A. U., \& Lopes de Oliveira, M. C. (2012). Diversidade e cultura da paz na escola: contribuições da perspectiva sociocultural. Porto Alegre: Editora Mediação.

Branco, A. U., \& Valsiner, J. (2012). Cultural psychology of human values. Charlotte, NC: Information Age Publishing.

Branco, A. U., Pessina, L., Flores, A., \& Salomão, S. (2004). A sociocultural constructivist approach to metacommunication in child development. In A. U. Branco \& J. Valsiner (Orgs.), Communication and metacommunication in human development (pp. 3-32). Greenwich, Connecticut: Information Age.

Brownell, C., \& Carriger, M. (1990). Changes in Cooperation and Self-Other Differentiation during the Second Year. Child Development, 61(4), 1164-1174. Doi:10.2307/1130884

Correa-Chávez, M. (2016). Cultural patterns of collaboration and communication while working together among U.S. Mexican heritage children. Learning, Culture and Social Interaction, 11, 130-141.

Dawes, R.M. (1980). Social dilemmas. Annual Review of Psychology, 31, 169-193.

Deutsch, M. (2015). Cooperation, competition, and conflict. In P. Coleman, \& M Detsch (Eds.) Morton Deutsch: A Pioneer in Developing Peace Psychology. Springer Briefs on Pioneers in Science and Practice, vol 30 (pp. 47-70). Cham: Springer.

Glaveanu, V., Branco, A. U., \& Neves-Pereira, M. (2016). Creativity and prosocial values: Nurturing cooperation within the classroom. In: Beghetto \& Kaufman (Eds.), Nurturing creativity in the classroom (pp. 287-307). Nueva York: Cambridge University Press.

Hay, D., \& Pawlby, S. (2003). Prosocial development in relation to children's and mothers' psychological problems. Child Development, 74(5), 1314-1327.

Hermans, H. (2001). The dialogical self: Toward a theory of personal and cultural positioning. Culture \& Psychology, 7, 243-281. 
Kagan, S., \& Madsen, M. C. (1971). Cooperation and competition of Mexican, Mexican-American, and Anglo-American children of two ages under four instructional sets. Developmental Psychology, 5(1), 32-39.

Lordelo, E. R., \& Carvalho, A. M. A. (2006). Padrões de parceria social e brincadeira em ambientes de creches. Psicologia em Estudo (Maringá), $11,99-108$.

Lotman, J. (2005). On the semiosphere. Sign Systems Studies, 33(1), 5-23.

Molleman, L., \& Gächtera, S. (2018). Societal background influences social learning in cooperative decision making. Evolution and Human Behavior, 39(5), 547-555.

Maciel, D., Branco, A., \& Valsiner, J. (2004). Bidirectional process of knowledge construction in teacher-student transaction. In A. U. Branco \& J. Valsiner (Orgs.), Communication and metacommunication in human development (pp. 109-125). Greenwich, Connecticut: Information Age.

Marsico, P., \& Varzi, A. (2015). Psychological and social borders: Regulating relationships. In J. Valsiner, G. Marsico, N. Chaudhary, T. Sato, and V. Dazzani (eds.), Psychology as the science of human being (pp. 327-336). Berlín: Springer-Verlag.

Matusov, E., Bell, N., \& Rogoff, B. (2002). Schooling as cultural process: Working together and guidance by children from schools differing in collaborative practices. Advances in Child Development and Behavior, 29, 129-160.

Palmieri, M., \& Branco, A. (2014). Educação infantil e cooperação. Londrina: Editora da Universidade Estadual de Londrina.
Pedrosa, A., \& Carvalho, A. M. A. (2006). Análise qualitativa de episódios de interação: uma reflexão sobre procedimentos e formas de uso. Psicologia: Reflexão e Crítica, 18(3), 431-442.

Pletzer, J., Balliet, D., Joireman, J., Kuhlman, M., Voelpel, S., \& Van Lange, P. (2018). Social Value Orientation, Expectations, and Cooperation in Social Dilemmas: A Meta-analysis. European Journal of Personality, 32, 62-83. Doi: 10.1002/ per.2139.

Rengifo-Herrera, F., \& Branco, A. (2014). Values as a Mediational System for Self-Construction: Contributions from Cultural Constructivism. Psicología desde el Caribe, 31(2), 304-326.

Salvatore, S., \& Valsiner, J. (2010). Between the General and the Unique: Overcoming the Nomothetic versus Idiographic Opposition. Theory \& Psychology, 20(6), 817-833.

Tacca, M. C. (2000). Ensinar e aprender: análise de processos de significação na relação professor $\mathrm{x}$ aluno em contextos estruturados. (Tese de doutorado não publicada. Universidade de Brasilia, Brasil).

Tateo, L. (2018). Affective semiosis and affective logic. New Ideas in Psychology, 48, 1-11.

Valsiner, J. (2014). An invitiation to cultural psychology. Londres: SAGE.

Valsiner, J., \& Rosa, A. (2007). The Cambridge Handbook of Sociocultural Psychology. Nueva York: Cambridge University Press.

Valsiner, J., Branco, A. U., \& Dantas, C. (1997). Co-construction of human development: Heterogeneity within parental belief orientations. In J. E. Grusec \& L. Kuczynski (Orgs.), Parenting and children's internalization of values (pp. 283-304). Nueva York: Wiley.

\section{Recibido: junio 5, 2019 Aprobado: agosto 29, 2019}

